\title{
Sarcomatous Hepatocellular Carcinoma with Remittent Fever
}

\author{
Yuji Inagaki ${ }^{1,2}$, Kazushi Sugimoto ${ }^{1}$, Katsuya Shiraki ${ }^{1}$, Naohiko Yoshizawa ${ }^{2}$, \\ Masahiko Tameda ${ }^{1}$, Suguru Ogura ${ }^{1}$, Misao Yoneda ${ }^{1}$, Yoshiyuki Takei ${ }^{1}$, Hiroyuki Fuke ${ }^{2}$, \\ Akira Hashimoto $^{2}$, Norihiko Yamamoto ${ }^{1}$ and Atsuya Shimizu ${ }^{2}$
}

\begin{abstract}
We herein report a rare case of hepatocellular carcinoma (HCC) with sarcomatous changes. A 66-year-old man was admitted to our hospital with a high fever and upper abdominal pain. Initially, he was diagnosed as having a liver abscess; however, antibiotic treatment and drainage were ineffective. Further imaging studies revealed the typical appearance of HCC: the tumor had invaded the hepatic and portal veins. Surgical resection of the tumor was performed. A pathological examination demonstrated the presence of a sarcomatous hepatocellular carcinoma. Sarcomatous hepatocellular carcinoma with remittent fever is a rare disease entity.
\end{abstract}

Key words: sarcomatous hepatocellular carcinoma, remittent fever, paraneoplastic syndrome

(Intern Med 51: 3025-3029, 2012)

(DOI: 10.2169/internalmedicine.51.7421)

\section{Introduction}

Generally, hepatocellular carcinoma (HCC) is asymptomatic. Fatigue, abdominal distention and low-grade fevers are sometimes observed with $\mathrm{HCC}$ due to a deterioration of the liver function. Fever as a primary symptom of HCC is very rare. We herein report a case of HCC involving a high fever and a high C-reactive protein (CRP) level that was initially diagnosed as a liver abscess.

\section{Case Report}

A 66-year-old Japanese man whose hepatitis $\mathrm{C}$ virus had been curatively treated was admitted to our hospital due to epigastralgia and a fever of two weeks' duration. On examination, the patient was found to be $171 \mathrm{~cm}$ tall and weighed $75 \mathrm{~kg}$. His temperature fluctuated between $36.4^{\circ} \mathrm{C}$ and $38.4^{\circ} \mathrm{C}$.

Table shows the laboratory data obtained on admission. A hypoechoic tumor measuring $4.4 \mathrm{~cm}$ in diameter was detected in two segments of the liver using ultrasonography.
Contrast-enhanced computed tomography (CT) revealed an irregularly demarcated mass with a maximum diameter of $55 \mathrm{~mm}$ in the S2 lobe with slight peripheral enhancement. The patient was diagnosed as having a liver abscess due to the high fever and elevated CRP level, although the CT findings did not have a distinctive pattern. First, therapy with intravenous antibiotics was initiated. Then, percutaneous drainage was performed; however there was no exudate. The patient remained febrile, even after undergoing these treatments. Meropenem was then administered intraarterially, and intravenous or intra-arterial antibiotics were administered for three weeks total; however, the fever continued and the CRP level rose to $18 \mathrm{mg} / \mathrm{dL}$.

Therefore, further evaluations were conducted. The levels of tumor markers at that time were as follows: alfa fetoprotein (AFP): $559 \mathrm{ng} / \mathrm{mL}$, AFP-L3 fraction: $51.3 \%$ and protein induced by vitamin $\mathrm{K}$ absence or antagonist II (PIVKA II): $78 \mathrm{mAU} / \mathrm{mL}$. A needle biopsy was performed; however the tissue was found to be necrotic. Sonazoid-enhanced ultrasonography detected a vessel flowing in the tumor in the early phase that was not enhanced in the late phase (Fig. 1). Angiography and CT under angiography revealed a large he-

${ }^{1}$ Department of Gastoenterology, Mie University School of Medicine, Japan and ${ }^{2}$ Department of Internal Medicine, Saiseikai Matsuzaka General Hospital, Japan

Received for publication January 28, 2012; Accepted for publication July 18, 2012

Correspondence to Dr. Katsuya Shiraki, katsuyas@clin.medic.mie-u.ac.jp 
Table. Laboratory Data on Admission

\begin{tabular}{|c|c|c|c|c|c|}
\hline WBC & 8200 & $/ \mathrm{mm}^{3}$ & $\gamma$-GTP & 37 & $\mathrm{IU} / \mathrm{L}$ \\
\hline $\mathrm{RBC}$ & $4.21 \times 10^{4}$ & $/ \mathrm{mm}^{3}$ & Amy & 52 & IU/L \\
\hline $\mathrm{Hb}$ & 11.8 & $\mathrm{~g} / \mathrm{dL}$ & $\mathrm{Cr}$ & $\begin{array}{l}10.0 \\
0.70\end{array}$ & $\begin{array}{l}\mathrm{mg} / \mathrm{dL} \\
\mathrm{mg} / \mathrm{dL}\end{array}$ \\
\hline HCT & 36.9 & $\%$ & CRP & 12.5 & $\mathrm{mg} / \mathrm{dL}$ \\
\hline PLT & $12.5 \times 10^{4}$ & $/ \mathrm{mm}^{3}$ & Glu & 122 & $\mathrm{mg} / \mathrm{dL}$ \\
\hline \multirow[t]{2}{*}{$\mathrm{PT}$} & 70 & $\%$ & & & \\
\hline & & & $\mathrm{HBsAg}$ & $(-)$ & \\
\hline TP & 8.2 & $\mathrm{~g} / \mathrm{dL}$ & HCVAb & $(+)$ & \\
\hline Alb & 3.2 & $\mathrm{~g} / \mathrm{dL}$ & HCVRNA & $(-)$ & \\
\hline T.Bil & 0.7 & $\mathrm{mg} / \mathrm{dL}$ & & & \\
\hline AST & 15 & IU/L & & & \\
\hline ALT & 17 & IU/L & & & \\
\hline LDH & 152 & IU/L & & & \\
\hline ALP & 264 & IU/L & & & \\
\hline
\end{tabular}

patic tumor measuring $5 \mathrm{~cm}$ in diameter in the left hepatic lobe with a tumor thrombus extending into the inferior vena cava (IVC) through the left hepatic vein (Fig. 2). The patient was diagnosed with $\mathrm{HCC}$ of the left hepatic lobe with a tumor thrombus extending into the supraphrenic IVC. Left hepatectomy with partial resection of the IVC and intravascular tumor thrombectomy were performed. The center of the mass was found to be almost completely necrotic. Histologically, the tumor primarily consisted of thickly proliferated spindle cells that reflected a sarcomatous change. Adjacent to the sarcomatous component, moderately to poorly differentiated HCC was observed (Fig. 3) (im(-), eg, fc(+), fc-inf(+), s0, n0, vp1, vv3, va0, b0, p0, sm(-, $300 \mu \mathrm{m}), \mathrm{CH}$, f3, pT3N0M0 Stage III). The noncancerous region of the liver exhibited severe fibrosis bridging the portal area. However, regenerative nodules were clearly formed that were histologically diagnosed as F3 according to the METAVIR score. Immunohistochemical staining was performed. The sarcomatous component was found to be positive for vimentin and pankeratin (AE1/AE3), slightly positive for cytokeratin 7 negative for cytoleratin 19 and cytokeratin 20 (Fig. 4). After tumor resection was performed, the remittent fever resolved and the serum CRP level returned to the normal range.

CT revealed mediastinal and portal hepatic lymphadenopathy. Paraesophageal lymph node metastasis of sarcomatous HCC was diagnosed using endoscopic ultrasonographyguided fine-needle aspiration. Chemotherapy with tegaful/gimeracil/oteracil potassium (S-1) and radiotherapy were administered; however the lymphadenopathy progressed. The chemotherapy regimen was then changed from S-1 to sorafenib. The progression of the lymphadenopathy continued; therefore, lymphadenectomy of the paraesophageal and retroperitoneal lymph nodes was performed 19 months after hepatectomy. The resected lymph nodes exhibited the same histologic characteristics as the liver tumor described above. The patient died of rupture of the para-esophageal lymph node into the esophagus 26 months after undergoing hepatectomy.

\section{Discussion}

Fever has long been recognized to be a symptom of malignant disease. Malignant diseases with pyrexia include malignant lymphoma, acute leukemia, sarcoma and renal cell carcinoma. Generally, HCC is often asymptomatic. Fatigue, abdominal distention and low-grade fever are sometimes observed with HCC due to a deterioration of the liver function. A high-grade fever as a primary symptom of HCC is extremely rare.

Okuda et al. (1) reported the cases of five patients with HCC who presented with pyrexia and leukocytosis that mimicked liver abscesses and concluded that the fevers could have been caused by a pyrogen produced by malignant cells or macrophages in response to necrosis. Yeh et al. (2) reported that $\mathrm{HCC}$ is a pyogenic liver abscess, and suggested that the pathogenesis of HCC involves either spontaneous liquefied necrosis of the tumor interior or biliary obstruction caused by tumor fragments. Masaki et al. (3) reported a similar case of HCC involving central necrosis due to rapid growth that had impaired the intrahepatic biliary duct and resulted in an infection of the tumor.

Fever is the most common symptom of paraneoplastic syndrome. The pathophysiology of paraneoplastic syndrome is complex and intriguing. Antibodies to tumor cells may cause a paraneoplastic disorder. In other cases, paraneoplastic syndrome results from humoral factors produced by cancer cells. Interleukin-8 (IL-8), interleukin-6 (IL-6) and granulocyte-colony stimulating factor (G-CSF) have been reported to be humoral factors related to fevers associated with HCC.

Akiba et al. (4) reported the expression of IL-8 in HCC to be accompanied by rapid tumor growth and a poor prognosis. Wigmore et al. (5) reported that the presence of IL-8 results in dose-dependent increases in the CRP level. HCC patients with a high IL-8 expression have a significantly higher frequency of venous invasion, portal vein invasion and bile duct invasion than HCC patients with a low IL-8 expression (4). Patients with G-CSF-producing HCC commonly have a very poor prognosis with rapid tumor growth and a high probability of distant metastasis (6-8). It has been reported that coproduction of G-CSF and IL-6 is associated with the production of IL-1, a known inflammatory cytokine, in G-CSF-producing cancer cell lines (9). IL-6 is considered to act as an endogenous pyrogen $(10,11)$ that regulates the synthesis of acute phase proteins, including CRP (12). HCC that produces humoral factors exhibits more rapid tumor growth and has a higher probability of distant metastasis.

A small number of cases of sarcomatous HCC with concomitant pyrexia have so far been reported $(1,13)$. The incidence of accompanying sarcomatous changes in HCC is relatively low: in one study of 350 HCC autopsies, sarcomatous changes were found in only $3.9 \%$ of these cases (14). In most cases of HCC with a sarcomatous appearance, the 

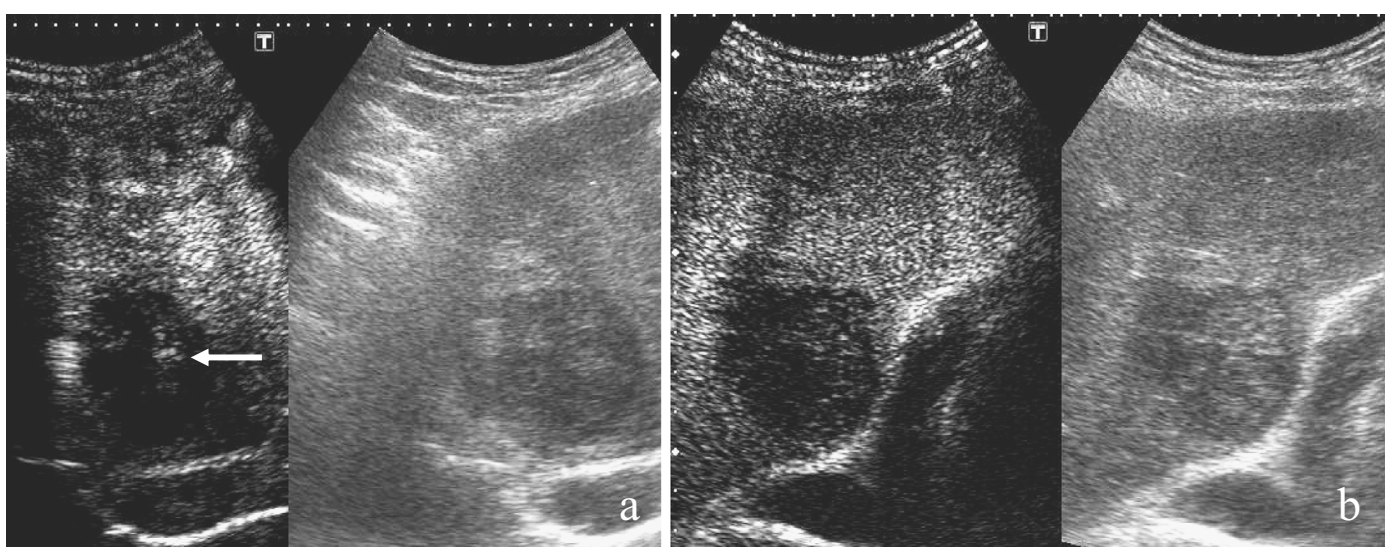

Figure 1. Sonazoid-enhanced ultrasonography. a) The inflowing artery in the early vascular phase (arrow). b) The tumor was not enhanced in the post-vascular phase.
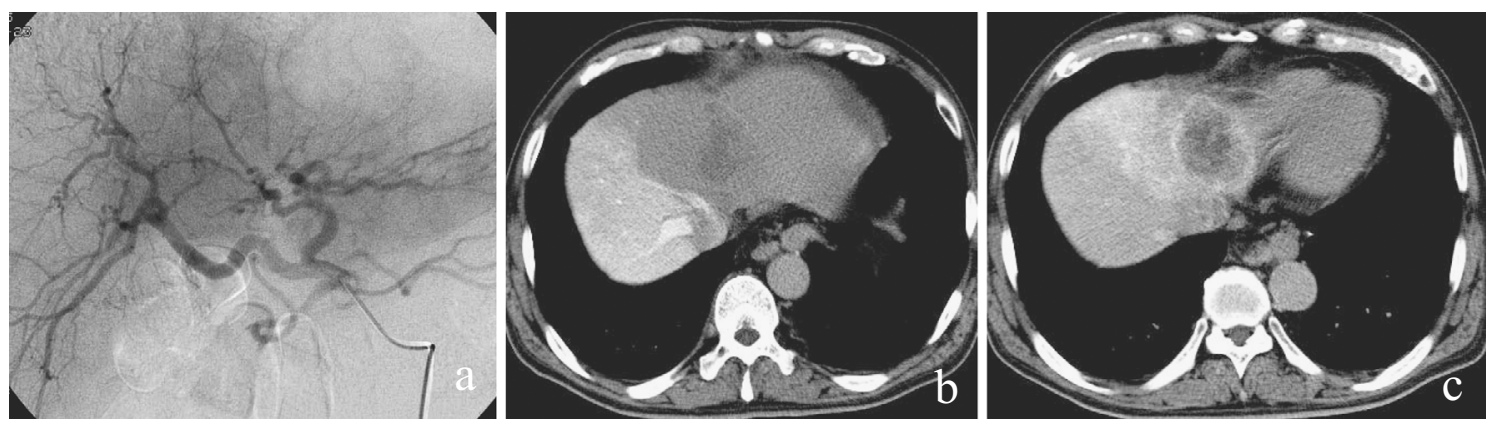

Figure 2. Angiographic findings and computed tomography (CT) under angiography. a) No tumor stains were observed. b) CT under superior mesenteric artery (SMA) angiography revealed a lack of left portal vein enhancement. c) CT under hepatic angiography revealed marginal, irregular, low-intensity enhancement of the tumor and a tumor thrombus from the left hepatic vein to the inferior vena cava.

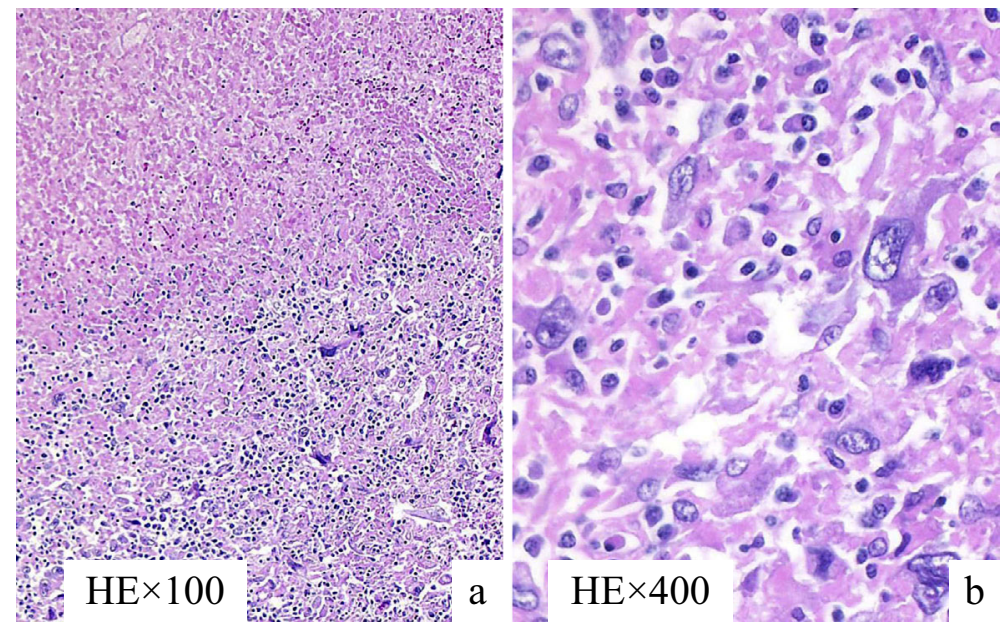

Figure 3. Microscopic findings of the resected specimen. a) Hematoxylin and Eosin (H\&E) staining, $\times 100$ magnification. b) $\mathbf{H} \& \mathrm{E}$ staining, $\times 400$ magnification. A poorly differentiated, compact type, sarcomatoid hepatocellular carcinoma.

serum AFP levels have been reported to be low or negative and the incidence of extrahepatic metastasis has been reported to be higher than that observed in usual HCC (14-17). Sarcomatous HCC reportedly shows invasive and sinusoidal growth patterns without fibrous capsules (14). This could be a reason for the high incidence of extrahepatic metastases in cases of sarcomatous HCC. Sarcomatous HCC is known to exhibit central necrosis and hemorrhage 


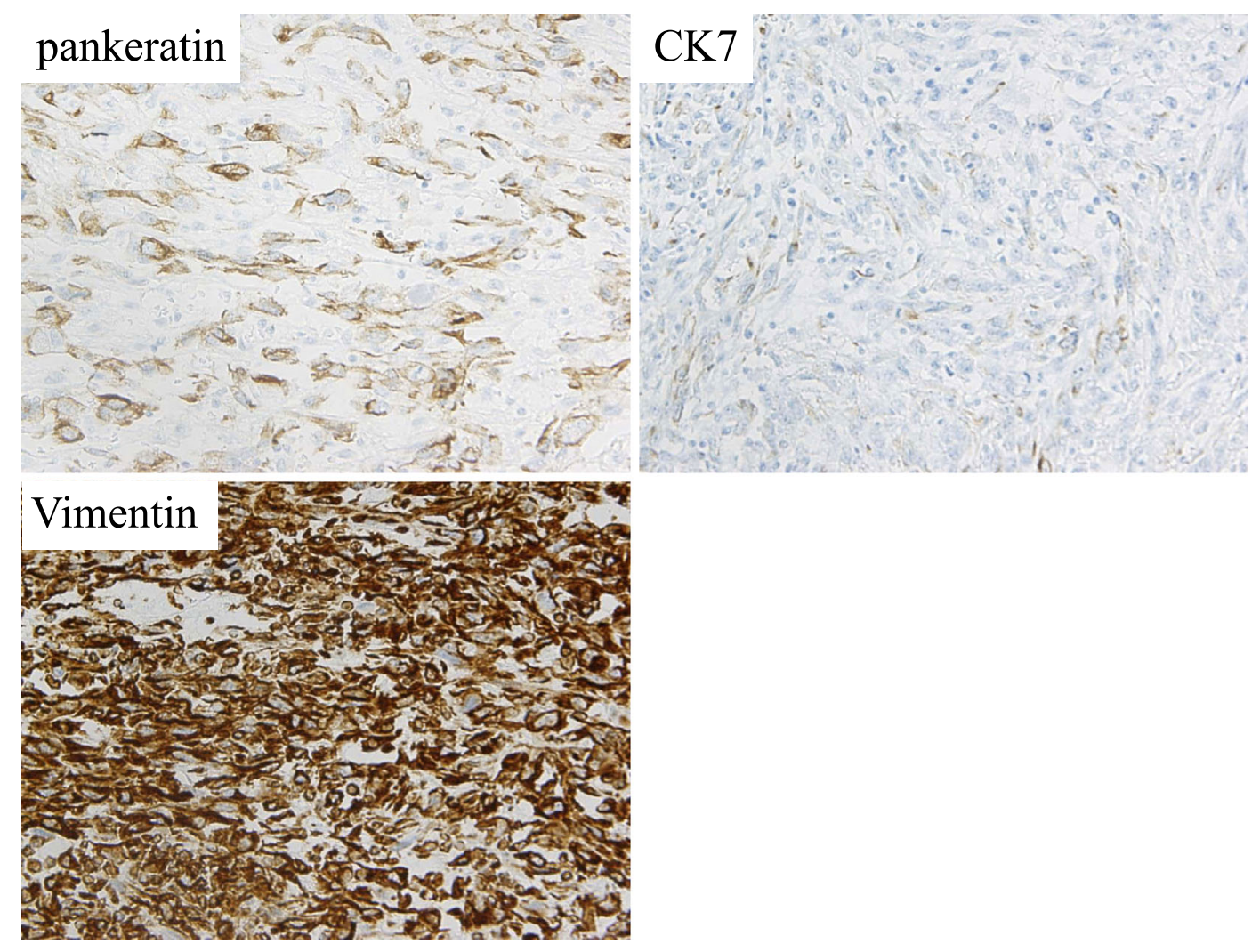

Figure 4. Immunohistochemical staining. The sarcomatous components stained positive for pankeratin ( $\times 200$ magnification), slightly positive for cytokeratin $7(\times 200$ magnification) and strongly positive for vimentin $(\times 200$ magnification).

more frequently than the more common types of HCC. Since sarcomatous HCC consists of very poorly differentiated cells and grows rapidly, it is conceivable that the neovasculature cannot adequately nourish the fast-growing malignant cells, which leads to necrosis (1). This feature has been clearly demonstrated on CT. In the present case, the periphery of the tumor exhibited ring-like enhancement and was shown to consist of viable cancerous tissue with a fibrous stroma. The imaging findings of the internal unenhanced region corresponded primarily with coagulation necrosis and hemorrhage. Such enhancement patterns on enhanced CT can represent metastases, intrahepatic cholangiocarcinomas or liver abscesses. In this case, the patient had a high fever and elevated CRP levels, and a hypovascular region was observed on enhanced CT. Therefore, a liver abscess was initially diagnosed. However, it is possible that GCSF or one of the other humoral factors described above was produced by the tumor and caused the fever and elevation of the CRP level observed in the present case, although this was not revealed on immunohistochemistry or by measuring the serum cytokine levels.

For treating sarcomatous HCC, a surgical resection should be considered because this type of tumor is resistant to transarterial embolization therapy (16). This type of tumor is also associated with a very poor prognosis due to its rapid growth and quite low resectability (18). Kakizoe et al. (14) reported that the mean survival time from the appearance of initial symptoms is 4.7 months. Regarding the surgical prog- nosis, Maeda et al. (19) reported that eight of 13 patients with surgically resected cases of sarcomatous HCC died of the disease within one year of undergoing surgery. The effects of chemotherapy on sarcomatous HCC remain uncertain. The SHARP Investigators Study Group (20) showed that treatment with sorafenib prolongs the median survival time and the time to progression by nearly three months in patients with advanced HCC. In the present case, chemotherapy with sorafenib was administered.

The present patient survived for more than two years from the initial appearance of symptoms. The relatively long survival time could have occurred, because the tumor was completely resected with no intrahepatic or distant metastases. In addition, the patient received multidisciplinary therapy (chemotherapy, radiotherapy, and lymphoadenectomy) after the recurrence of the sarcomatous HCC. In conclusion, we herein described a case of sarcomatous HCC involving remittent fever that was initially diagnosed as a liver abscess.

The authors state that they have no Conflict of Interest (COI).

\section{References}

1. Okuda K, Kondo Y, Nakano M, et al. Hepatocellular carcinoma presenting with pyrexia and leukocytosis: report of five cases. Hepatology 13: 695-700, 1991.

2. Yeh TS, Jan Y-Y, Jeng LB, Chen TS, Hwang TL, Chen MF. Hepa- 
tocellular carcinoma presenting as pyogenic liver abscess: characteristics, diagnosis, and management. Clin Infect Dis 26: 12241226, 1998.

3. Masaki Y, Ueno T, Hamada H, Akiyama T. A case of hepatpcellular carcinoma with sarcomatous change that occurred in a patient with chronic hepatitis $\mathrm{C}$ which was difficult to distinguish from liver abscess. Jpn J Gastroenterol Surg 40: 1490-1495, 2007 (in Japanese, Abstract in English).

4. Akiba H, Yano H, Ogasawara S, Higaki K, Kojiro M. Expression and function of interleukin-8 in human hepatocellular carcinoma. Int J Oncol 18: 257-264, 2001.

5. Wigmore SJ, Fearon KC, Maingay JP, Lai PB, Ross JA. Interleukin- 8 can mediate acute-phase protein production by isolated human hepatocytes. Am J Physiol 273: 720-726, 1997.

6. Araki K, Kishihara F, Takahashi K, et al. Hepatocellular carcinoma producing a granulocyte colony stimulating factor: report of a resected case with a literature review. Liver Int 27: 716-721, 2007.

7. Aita K, Seki K. Carcinosarcoma of the liver producing granulocyte-colony stimulating factor. Pathol Int 56: 413-419, 2006.

8. Joshita S, Nakazawa K, Koike S, et al. A case of granulocytecolony stimulating factor-producing hepatocellular carcinoma confirmed by immunohistochemistry. J Korean Med Sci 25: 476-480, 2010.

9. Suzuki A, Takahashi T, Okuno Y, et al. IL-1 production as a regulator of G-CSF and IL-6 production in CSF-producing cell lines. Br J Cancer 65: 515-518, 1992.

10. Dinarello CA. Cytokines as endogenous pyrogens. J Infect Dis 179: 294-304, 1999.
11. Luheshi GN. Cytokines and fever. Ann NY Acad Sci 856: 83-89, 1998.

12. Castell JV, Gomez-Lechon MJ, David M, et al. Interleuki-6 is the major regulator of acute phase protein synthesis in adult human hepatocytes. FEBS Lett 242: 237-239, 1989.

13. Hayashi T, Honda H, Kaneko K, et al. Hepatocellular carcinoma with pyrexia: report of a case. Radiation Med 13: 133-136, 1995.

14. Kakizoe S, Kojiro M, Nakashima T. Hepatocellular carcinoma with sarcomatous change: clinicopathologic and immunohistochemical studies of 14 autopsy cases. Cancer 59: 310-316, 1987.

15. Sugihara S, Kakizoe S, Ito Y, Nakashima O, Maruiwa M, Kojiro M. Clinicopathologic study of hepatocellular carcinoma with sarcomatous appearance. Kanzo (Acta Hepatol Jpn) 29: 71-76, 1988 (in Japanese, Abstract in English).

16. Ishii M, Abe M, Hirai Y, et al. The clinical study of hepatocellular carcinoma with sarcoma-like feature. Kanzo (Acta Hepatol Jpn) 29: 734-741, 1988

17. Honda H, Hayashi T, Yoshida K, et al. Hepatocellular carcinoma with sarcomatous change: characteristic findings of two phased incremental CT. Abdom Imaging 21: 37-40, 1996.

18. Tsujimoto M, Aozasa K, Nakajima Y, Kariya M. Hepatocellular carcinoma with sarcomatous proliferation showing an unusual and wide-spread metastasis. Acta Pathol Jpn 34: 839-845, 1984.

19. Maeda T, Adachi E, Kajiyama K, Takeneka K, Sugimachi K, Tsuneyoshi M. Spindle cell hepatocellular carcinoma. A clinicopathologic and immunohistochemical analysis of 15 cases. Cancer 77: 51-57, 1996.

20. Llovet JM, Ricci S, Mazzaferro V, et al. Sorafenib in advanced hepatocellular carcinoma. N Engl J Med 359: 378-390, 2008.

(C) 2012 The Japanese Society of Internal Medicine http://www.naika.or.jp/imonline/index.html 